\title{
Observing Galactic Be/X-ray binaries with the SALT high-resolution spectrograph
}

\section{L.J. Townsend*}

Astrophysics, Cosmology and Gravity Centre, Department of Astronomy, University of Cape

Town, Private Bag X3, Rondebosch 7701, South Africa

E-mail: townsendeast.uct.ac.za

\section{V.A. McBride}

Astrophysics, Cosmology and Gravity Centre, Department of Astronomy, University of Cape Town, Private Bag X3, Rondebosch 7701, South Africa; South African Astronomical Observatory, PO Box 9, Observatory 7935, South Africa

\section{S.M. Crawford}

South African Astronomical Observatory, PO Box 9, Observatory 7935, South Africa

\section{Schwab}

Department of Astronomy \& Astrophysics, The Pennsylvania State University, 525 Davey Lab, University Park, PA 16802, USA

\begin{abstract}
The measurement of the orbital parameters of Be/X-ray binaries is central to our understanding of their formation and observed multi-wavelength variability. The typically long and moderately eccentric orbits make unlocking this information from X-ray pulse timing difficult, as the observer must wait for a large X-ray outburst lasting most of a binary orbit to fit the necessary models. This has been done successfully for shorter period systems $\left(\mathrm{P}_{\text {orb }} \lesssim 30 \mathrm{~d}\right)$, but is rarely an option in longer period systems. In this paper, we describe recent efforts to overcome this observational bias by using optical radial velocity measurements of the companion stars of longer period Be/Xray binaries to calculate their orbital parameters. These results will significantly increase the number of long period systems with binary solutions and may help in determining the binary formation channel taken by these systems.
\end{abstract}

SALT Science Conference 2015

1-5 June 2015

Stellenbosch Institute of Advanced Study, South Africa

\footnotetext{
*Speaker.
} 


\section{Introduction}

High-mass X-ray binaries (HMXB) are prominent sources of variable X-ray emission in the local universe. They comprise a young, massive star and a compact object. The compact object accretes material from the massive star, emitting X-rays. These systems can be divided into two main categories: supergiant X-ray binaries (SGXRB) and Be/X-ray binaries (BeXRBs) [1]. The SGXRBs are typically characterised by a short $\left(\mathrm{P}_{o r b} \lesssim 10 \mathrm{~d}\right)$ orbit that has been circularised by the tidal influence of the supergiant star on the compact object. The compact object accretes persistently from the stellar wind of the companion, either spherically or via Roche-lobe overflow. The BeXRBs consist of a Be star secondary and almost exclusively a neutron star primary, though there is one example of a Be/Black hole system [2] and a small number of Be/White dwarf systems [3]. They are typically characterised by longer orbits $\left(\mathrm{P}_{\text {orb }} \sim 10-500 \mathrm{~d}\right)$ with a range of possible eccentricities ( $\mathrm{e} \sim 0.05-0.90)$ [4]. It is these systems that are the subject of this paper.

The non-zero eccentricity in BeXRBs mean that the X-ray emission we see is transient in nature and occurs only when the neutron star is close enough to the circumstellar material around the Be star to accrete (usually around periastron) or when the circumstellar disc increases in size to engulf the neutron star. These are the classical descriptions of Type I and Type II outbursts, respectively. Type I outbursts are modulated on the orbital period of the system, whilst Type II outbursts can occur at any orbital phase and typically last much longer and at a greater luminosity than Type I outbursts. The orbital modulation present in Type I outbursts allows the orbital period to be derived through timing analysis of the resultant light curve, though the other binary parameters (eccentricity, epoch of periastron, longitude of periastron and projected semi-major axis) can only be determined precisely through modelling the neutron star pulse period evolution in Type II outbursts.

As described above, the binary parameter space occupied by the BeXRBs is large and due to the nature of the outbursts we observe, only the orbital period and neutron star spin period have been determined for a significant proportion of the population. The remaining binary parameters are still only known for a fraction of the population [4]. The good understanding we have of the distribution of the spin and orbital periods has lead to two important discoveries. Firstly, there is a positive correlation between the two periods, albeit with a fairly large scatter [5]. Secondly, the distribution of both periods is bimodal [6]. This has been attributed to the formation channel being different for the two sub-groups; namely forming through two different types of supernova. This theory has since been countered by work suggesting that the distribution is down to different accretion modes of the neutron star [7]. It is clear that regardless of which theory is correct in describing the distribution seen, the extended binary parameter space of BeXRBs holds important information to understand their nature. Our focus here is on the distribution of eccentricities amongst the population and the relationship between the eccentricity and the binary period. Currently, the large majority of systems studied reside in the short orbit, moderate eccentricity part of the parameter space [4] and so drawing conclusions about the population from the distribution is difficult. Determining whether this is an observational bias or whether there is a particular correlation or distribution in the data will require the calculation of eccentricities in more long period binary systems. As already described, this is very difficult to achieve with X-ray data and very time-consuming using optical radial velocity observations. 


\begin{tabular}{|l|c|c|c|c|c|}
\hline Source & $\mathrm{P}_{\text {spin }}(\mathrm{s})$ & $\mathrm{P}_{\text {orb }}(\mathrm{d})$ & Eccentricity & axsini (lt-s) & Spectral Type \\
\hline 4U 0728-25 & 103.2 & 34.5 & $?$ & $?$ & O8-9Ve \\
4U 1036-56 & 862 & 61.0 & $?$ & $?$ & B0Ve \\
1A 1118-61 & 407.7 & 24.0 & $<0.16$ & 54.9 & O9.5Ve \\
4U 1145-61 & 292.4 & 186.5 & $>0.5$ & $?$ & B1Ve \\
\hline
\end{tabular}

Table 1: Spin period, orbital period and spectral type of the four sources under observation. The aim is to measure the eccentricity and semi-major axis for each source and place a constraint on the neutron star mass for 1A 1118-61 using the known X-ray mass function.

In this paper, we describe our programme to begin increasing the number of long-period BeXRB systems with well determined binary parameters using long-term monitoring observations with SALT/HRS. We discuss our plans to use this new information to help understand the parameter distribution and what this may teach us about the nature of this type of binary system.

\section{SALT/HRS monitoring of Galactic BeXRBs}

The new high-resolution spectrograph on the Southern African Large Telescope (SALT) was designed to have three resolution modes. We have used the low resolution mode ( $\mathrm{R} \sim 16000)$ to measure velocity amplitudes and eccentricities for a number of Galactic BeXRBs. The targets were chosen based primarily on their brightness and visibility to SALT, as well as there being no well constrained eccentricity. We also required the targets to have well defined and fairly long orbital periods and well defined spectral types. This information is summarised in Table 1. For the first three systems, we have acquired 10 spectra spaced evenly around the orbits in 0.1 phase spacing. The last system was added later in the multi-semester programme and has accrued 8 spectra spaced over approximately half of the orbit. This may hamper efforts to properly model the radial velocity in this system and may require a small number of subsequent observations at appropriate orbital phases in the future.

Basic CCD reductions including bias subtractions, gain corrections, and flat fielding were handled by the ccdproc package. Spectroscopic reductions of the data were carried out using the pyhrs package. The software creates an order frame from a flat field image that assigned each pixel in the image to a specific order. Prior to extraction, each order was corrected for spatial and spectral curvature. To calculate the wavelength solution, a spectrum was extracted by summing the rows in the order. The resultant spectrum was then passed to the specidentify task in the PySALT reduction package [8] for wavelength identification and calculation of the wavelength solution. Next, the target spectrum was extracted from our observations. The extraction of the order was performed as described with the final spectrum being a result of a flux-weighted co-addition of all of the illuminated rows in the order and the wavelength derived from the solution calculated for that order.

Figure 1 shows some preliminary data from this programme and are representative of the quality of the lines seen in most spectra obtained. The data are currently in the final stages of analysis, including the refinement of the wavelength solutions, before modelling of the radial velocities can begin. The data will also be used as part of a secondary project to measure the orbital variability of the Balmer emission lines in each system, though this will be discussed fully in a separate paper. 

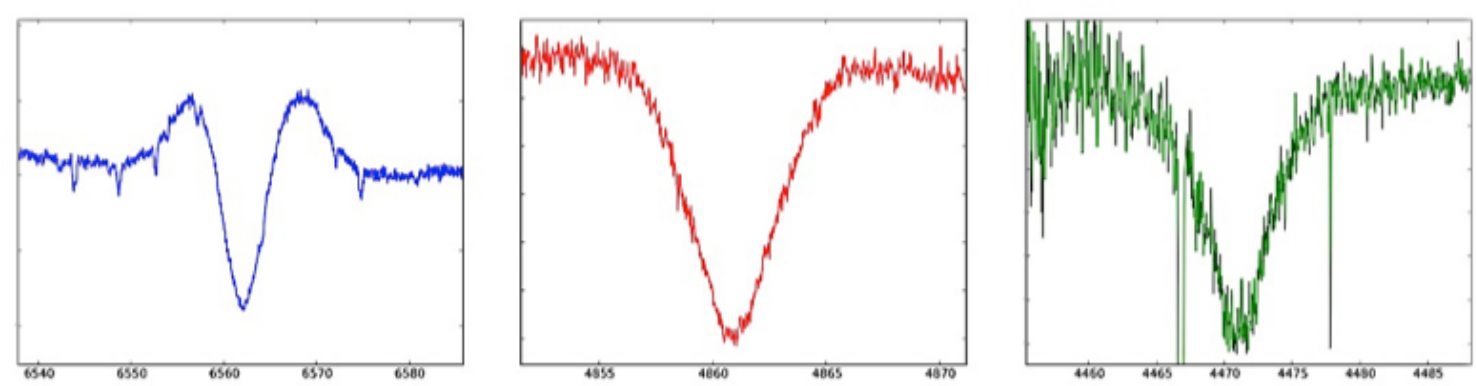

Figure 1: Example $\mathrm{H} \alpha, \mathrm{H} \beta$ and HeI 4471 line profiles of $4 \mathrm{U}$ 1036-56 taken with SALT/HRS. These profiles are representative of the quality of the lines seen in most spectra obtained to date. Measuring radial velocities from shifts in the HeI and Fe lines in these spectra will give us the eccentricity and optical mass function for each system.

\section{Implications and conclusions}

As mentioned at the beginning of this paper, the discovery of a bi-modality in the distribution of orbital periods of BeXRBs is proposed as tentative evidence of the population forming through two distinct channels of supernova: electron-capture and core-collapse (short and long period systems respectively). This two-channel formation theory is also proposed to explain the small, but distinct group of BeXRBs with near-circular orbits, as opposed to the majority that have moderate eccentricities [9]. These so-called 'low eccentricity transients' are located roughly in the shaded region in Figure 2. When these systems are excluded from Figure 2, there appears to be a log-linear trend between the eccentricity and orbital periods for all HMXBs. However, this is misleading, as the evolutionary sequence of SGXRBs almost certainly involves tidal circularisation and so these systems should be considered separately from the BeXRBs. When this is done, the parameter space for the BeXRBs is currently very messy. It is unclear whether these systems are uniform across the parameter space or whether there is a bimodality or other trend hidden in the data. The only observation one can currently make with a reasonable certainty is that there are likely no highly eccentric systems with a short period ( $\mathrm{P} \sim 10-20 \mathrm{~d})$, as this period space has been well sampled.

Placing the systems in Table 1 on Figure 2 will help determine whether the eccentricity distribution is continuous, bimodal or random and lead our ideas on the binary formation. Very little is known about the binary parameters of $4 \mathrm{U}$ 0728-25 and $4 \mathrm{U}$ 1036-56. These two systems have moderate periods and could have eccentricities placing them almost anywhere on Figure 2. There is a small group of systems around $\mathrm{P} \sim 30-40 \mathrm{~d}$ in Figure 2 with eccentricities of almost exactly the same value of 0.4. Should $4 \mathrm{U}$ 0728-25 deviate significantly from this value, it will be one of the first such systems in this period range and would lend support to the bimodal hypothesis. $4 \mathrm{U}$ 1036-56 is probably the most important system to model as there are very few others in this period range on Figure 2. Again, a very high or low eccentricity will support a bimodal eccentricity, whilst a more moderate value may support a continuous distribution.

1A 1118-61 was determined to have a $24 \mathrm{~d}$ orbital period and a low eccentricity through modelling of RXTE pulse arrival times [12]. This system was chosen to try and constrain the eccentricity to better than was allowed by the X-ray outburst data and to use the X-ray mass function and optical radial velocity to place limits on the binary component masses, given a range of possible 


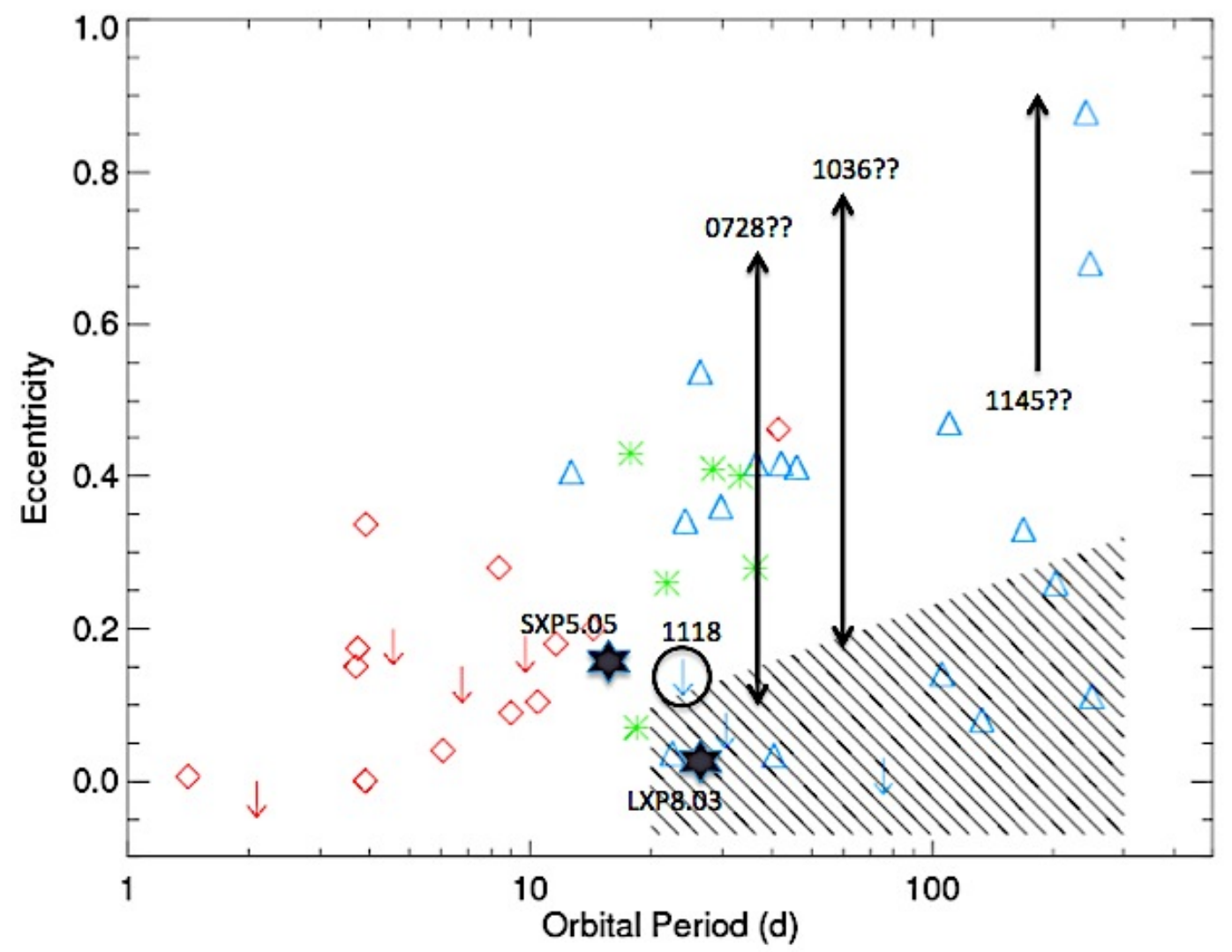

Figure 2: Orbital period vs. eccentricity plane for high-mass X-ray binaries adapted from [4]. The red diamonds are supergiant systems, the blue triangles are Galactic BeXRBs and the green stars are SMC BeXRBs. The black stars indicate the recent work of [10] and [11]. The black arrows and circle indicate where the 4 systems observed with SALT are anticipated to lie.

binary inclinations. There have been no dynamically determined masses in BeXRBs at the time of writing, and so placing limits on the masses in this system could prove an important addition to the small list of measured neutron star masses.

$4 \mathrm{U}$ 1145-61 was added to the programme later after the award of more observing time. It was chosen as it was reported to have a very high eccentricity from analysis of Ariel V X-ray data [13]. It is clear in Figure 2 that the systems with periods of 200-300 d have a large range in measured eccentricities. Very high eccentricities in the population as a whole are very rare, so a precise measurement of this system is crucial. Due to our current measurements only spanning around half an orbit, a small number of spectra may be required in future to fully constrain the radial velocity solution.

\section{Summary}

We have presented some preliminary results in our SALT/HRS long-term monitoring programme of Galactic BeXRBs. The data collection has been completed and analysis is at an early stage. We have seen the quality of the high resolution spectra obtained by looking at the strong 
Balmer emission lines and Helium I absorption lines present. The wavelength calibration of the new spectrograph is currently being refined, which will allow us to begin to model the radial motion of these binaries. Our goal is to use these data to begin to fill in the empty parts of the binary parameter space and use the distribution to help lead our ideas on how BeXRBs form. Even with the addition of these 4 systems, this is just a small step toward fully exploring this distribution. In future, large scale spectral monitoring programmes will be needed to gain a complete census of the binary parameter space.

\section{Acknowledgments}

LJT would like to thank the Claude Leon Foundation for support via their post-doctoral fellowship programme and the South African National Research Foundation for conference travel support.

\section{References}

[1] Reig P., 2011, Ap\&SS, 332, 1

[2] Casares J., et al., 2014, Nature, 505, 378

[3] Li K.L., et al., 2012, ApJ, 761, 99

[4] Townsend L.J., et al. 2011, MNRAS, 416, 1556

[5] Corbet R.H.D., 1986, MNRAS, 220, 1047

[6] Knigge C., et al., 2011, Nature, 479, 372

[7] Cheng Z.Q., et al., 2014, ApJ, 786, 128

[8] Crawford S.M., et al., 2010 SPIE, 7737, 25

[9] Pfahl E., et al., 2002, ApJ, 574, 364

[10] Coe M.J., et al., 2015, MNRAS, 447, 2387

[11] Kuehnel et al., 2014, ATel, 5856

[12] Staubert R., et al., 2011, A\&A, 527, 7

[13] Watson M.G., et al., 1981, MNRAS, 195, 197 